\title{
The Allocation of Business Model Components under Presence of Uncertainties by the Branch-and-Bound Method
}

\author{
Snežana Nestić (iD, ${ }^{1}$ Aleksandar Aleksić $\left(\mathbb{D},{ }^{1}\right.$ Jaime Gil Lafuente, ${ }^{2}$ Tijana Cvetić $\left(\mathbb{D},{ }^{1}\right.$ \\ and Goran Durić3 \\ ${ }^{1}$ University of Kragujevac, Faculty of Engineering, Kragujevac 34000, Serbia \\ ${ }^{2}$ University of Barcelona, Faculty of Economics and Business Science, Barcelona 08001-08042, Spain \\ ${ }^{3}$ Faculty of Mechanical Engineering, University of Belgrade, Belgrade 11120, Serbia \\ Correspondence should be addressed to Aleksandar Aleksić; aaleksic@kg.ac.rs
}

Received 9 December 2021; Revised 31 December 2021; Accepted 20 January 2022; Published 16 February 2022

Academic Editor: Darko Božanić

Copyright () 2022 Snežana Nestić et al. This is an open access article distributed under the Creative Commons Attribution License, which permits unrestricted use, distribution, and reproduction in any medium, provided the original work is properly cited.

As the only constant in business is change, business transformation is essential for adopting new perspectives and business trends. One of the keys to performing successful business transformation is to be fully aware of the current components of the business model. This research aims to allocate the business model components (BMCs) to defined business model components groups (BMCGs) by developing a new approach that integrates fuzzy sets and heuristic algorithms. The allocation results enable a comprehensive analysis of business model frameworks and give a good connection to research in the domain of strategic management and business process modeling. For allocation, the decision-makers (DMs) are employing the linguistic terms modeled by the fuzzy sets theory. The considered problem is stated as an integer programming model where the optimal solution is given by a $\mathrm{B} \& \mathrm{~B}$ algorithm. The model is tested on a sample of forty experts from four different economic sectors.

\section{Introduction}

During the last decades, defining business models has become a very important issue in the domain of business and management since a lot of scholars believe that a company's business can be presented through a business model. The term business model (BM) has been described by many authors appointing that it covers the architecture of product flows, services, and information, including the description of different business entities and their roles, as well as a description of potential benefits for different business entities and a description of the source of income [1]. BMs are used for determining the structure, relations, and success factors of an organization. They can serve as generators of competencies, especially in terms of rapid changes in the market. BMs describe how marketable information, products, and/or services are generated utilizing a company's value-added component. In addition to value creation, different components are taken into consideration to achieve generating and securing the competitive advantage. Those are related to strategic, customer, and market components. In literature, five different perspectives of this term can be found: business model activities, business model logics, business model archetypes, business model alignment, and business model components (BMCs) [2].

BMCs perspective is taken by authors who propose structuring BM based on its essential components to capture the important parts of the business and to create the operational framework. Several studies have investigated various definitions and lists of BMCs [3-5].

Stating the fact that BM should interpret the most significant segments of the business and the basic features of the enterprise, the most significant issue in this research area is defining the BMCs. For more efficient and effective management of the enterprise, a certain number of scholars denote the idea of allocating BMCs to BMCGs with the same purpose or some other attributes.

Awareness of BMCs and their organization in a business model is crucial for the business transformation and achieving the long-term sustainability of the company. This is highly applicable for the companies that are shifting their 
production to the requirements of industry 4.0 . The motivation for this research comes from the fact that there is no genuine set of BMCs in literature, nor is the allocation of BMCs to BMCGs performed in an exact way. Such allocation is highly dependent on the experience of decision-makers (DMs). At the same time, the wider audience is questioning the need for the business model development or BMs improvement, considering the legacy of established companies [6]. As a known management tool, an affinity diagram could be used for this purpose, although there is a certain level of ambiguity related to BMCs classification. The authors believe that the mentioned classification tool should be enhanced. This complies with the ongoing research trend that existing methodologies should be modified to address more complex situations [7].

By using the words of natural language, DMs can better express their assessment compared to the situation when they use real numbers. Linguistic expressions can be quantitatively described by using the fuzzy sets theory [8]. If fuzzy sets are employed as a tool for describing different variables, many literature sources stand for the application of type-1 fuzzy numbers [9-11] in many research fields.

Allocation of BMCs to BMCGs can be denoted as a medium-sized instances Integer Linear Programming (ILP) problem with a linear fitness objective function and a set of linear constraints. In literature, many exact methods address the problems of combinatorial optimization [12]. The Branch-and-Bound $(B \& B)$ method has been significantly employed for solving a variety of combinatorial optimization problems, for example, production planning problems $[13,14]$ and energy system problems $[15,16]$. It is worth mentioning that most software solutions dedicated to combinatorial optimization problems are based on the $B \& B$ method such as Gurobi 9.1 that is employed in the scope of this research.

In compliance with the stated, the objective of this research is to (1) define the appropriate BMCs that may be used for the constitution of the enterprise BM at the level of the considered industry, (2) model the existing uncertainties by using fuzzy sets theory, and (3) allocate BMCs to defined BMCGs by using an exact method, to help the corporate managers understand BMs and help them make strategic choices.

The paper is organized in the following way: Section 2 provides a detailed analysis of papers that can be found in literature covering different research domains, for example, a business model, modeling of uncertainty, and allocation domain. Section 3 describes the used methodology. In Section 4, the proposed model is tested based on real-life data. The discussion of the given results and conclusion are presented in Section 5.

\section{Literature Review}

This section introduces the wide analysis of relevant literature sources divided into two parts: (i) analysis of business model components and (ii) allocation problem under uncertainties by using the B\&B method.
2.1. Business Model Components. For the BCMs' allocation in this study, authors have analyzed many literature sources in which the BMCs have been stated. BMCs may be analyzed by considering mutual interaction between business subjects, creating values and income sources [17]. The significance of the economic component in BM is emphasized in the conducted research [18], as well as firms' economic dimension with components that correspond to the determinants of firms' profitability [19]. Some scholars [6] look at BMs at a general level and provide the ontology of BM by emphasizing the significance of the component, differentiation, and strategic control, which represent the economic need to differentiate and protect revenue streams.

To utilize the technology to increase the effectiveness of the company, the components that emphasize informational technologies are introduced into the BM [20, 21].

In studies $[22,23]$, the inclusion of value in the model as its component was used for the first time. The value proposition dimension might be enhanced with three components: competitors, key business components, and structure [24]. For an easier understanding of how the BM fulfilled a potent value proposition profitably, the business model framework was defined [25]. The authors defined their framework by placing components in four groups: Customer Value Proposition, Profit Formula, Key Resources, and Key Processes. The Key Resources group put focus on the key components that create value for the customer and the company and, unlike other authors, further introduced components of equipment and brand. The Key Processes group also included rules, metrics, and norms. A consolidated view of the components [23] might be based on the value proposition (the offering, the target customer, and the basic strategy), the value creation and delivery system (resources and capabilities, organization, and position in the value network), and the value capture (revenue sources and the economics of the business).

As a component of BMs, trading mechanisms, trading protocols was introduced with increased employment of dynamics of electronic commerce [26]. The more comprehensive research in the domain of e-commerce models has resulted in the introduction of a new component entitled product innovation [27]. The authors then identified 9 of the most common BMCs [28]. They included all the components related to competition and implementation of BM. Considering that these components, although they are connected with the BM, are not their internal part, the authors introduced a component delivery channel.

The component entitled goods and services production and exchanges was introduced through the presentation of an analytical framework for comparing different BMs for producing information goods and digital services [29].

The review of literature on BMs in the contexts of technological, organizational, and social innovation brought more BMs components [30]. They proposed components including value proposition, supply chain, customer interface, and financial model that BMs should meet to be sustainable. The business model framework was analyzed from the sustainability perspective [1], so the following 
components were introduced for the first time: governance, process measure, and value configuration in the BM.

A new set of BM components had been developed based on BM innovations, and a new component entitled core competences was introduced [31].

One of the conducted research projects emphasized the heterogeneousness of contents of BM in literature, which manifested as $\mathrm{T}$ [32]. The authors had systematized all the components from literature and introduced the component procurement. Based on the detailed analysis of these studies, it can be said that the allocation was performed based on the assessment of the DMs and, to a great degree, depending on their knowledge and experience $[3,5,22]$. An affinity diagram can be used to categorize BM components found in literature [4].

\subsection{Allocation of BMCs Problem under Uncertainties.} This section is supplied with the literature review emphasizing the importance of linguistic variables modeling with FST and solving a variety of optimization problems in different research domains by the $\mathrm{B} \& \mathrm{~B}$ method.

2.2.1. Modeling by Using FST. A significant number of scholars stand beside the fact that it is suitable for DM to employ approximate information and uncertainty to generate decisions. The development of mathematics, especially probability theory and FST [8], has enabled the quantitative description of linguistic expressions. The application of a stochastic approach in the processing of uncertainty requires the existence of any relevant data records and a large complexity of computation. On the other hand, FST is a valuable tool that copes with two major problematic areas of the treated problem such as imprecision and ambiguity.

A fuzzy set is represented by its membership function, and the shape of the membership functions can be based on one's experience, the subjective belief of DMs, intuition, and contextual knowledge about the concept modeled [8]. The selection of membership function shapes can be treated as a problem itself. Many authors use trapezoidal fuzzy numbers (TrFNs) and triangular fuzzy numbers (TFNs). The range of maximum triangular membership must be around the crisp point of the triangle. The range of the maximum trapezoidal membership function is wider. Using TFNs decreases the complexity of calculations, and at the same time, the calculated results are accurate enough. In this research, modeling of judgments of the DMs is performed by TFNs.

The granularity depends on the treated problem size. Based on literature, the seven categories can be used at most. In this research, the five have been employed TFNs by analogy $[11,33]$.

The domain of fuzzy sets can be defined on different measurement scales, for instance, common measurement scale, $[1-5]$, or $[0-1]$ as in this research.

If the problem is presented as a fuzzy group decisionmaking problem, an aggregation procedure should be conducted $[8,34]$.
2.2.2. A Branch-and-Bound Method Analysis. The B\&B method is based on dividing the total set of feasible solutions into smaller subsets of solutions. These smaller subsets can be evaluated until the best solution is obtained. This exact method requires large computer resources to solve very large problems, and therefore a heuristic is required for most real problems. According to the B\&B method, the node with the smallest lower bound is extended at each iteration. If the number of decision variables is low, the $B \& B$ method is very useful and easily implemented to obtain the integer solution.

The problem of the assembly line design with parallel stations could be stated as ILP [35]. In this case, the objective function is defined as minimizing the number of stations respecting different conditions. A similar problem has been treated by [16]. Also, the energy-efficient management problem has been analyzed [36]. This problem is defined as a Mixed-Integer Linear Programming model. The objective function is defined as the maximization of the total capacity. The deviation of the variables to the operating point presents a constraint. The scheduling problem related to the jobs that may be realized at several machines is considered by [14]. The new $B \& B$ method is proposed, and it is tested on problems with 100 instances.

Another kind of problem that may be solved by B\&B is carpooling, which consists of defining the subsets of passengers that will share each vehicle and the routes that the drivers should follow. Several authors introduce the presumption that the vehicles and drivers are not known beforehand. Carpooling problem is treated by [15]. The objective function is formulated as minimizing three different costs. Constrains are given by using 14 linear equations. It has been shown that, by applying the B\&B method, the optimal solution for the problem is efficiently obtained. The optimal design of energy supply systems in consideration of multiperiod operation is formulated as a MixedInteger Linear Programming task by [37]. The objective function is defined as the sum of the annual capital cost of equipment, the annual demand charge of utilities, and the annual energy charge of utilities per hour at each period. The optimal solution is found from the condition when the goal function reaches a minimum while satisfying all constraints.

One important application of the $\mathrm{B} \& \mathrm{~B}$ method is presented in the domain of inventory management [13]. In the presented model, the objective function is defined as integrated profit. The optimal solution is found when the goal function reaches the maximum value while satisfying the set cost limits.

\section{The Methodology}

In this section, the hybrid model, which integrates panel discussion, fuzzy sets theory, ILP, and Branch-and-Bound algorithm, is presented. The proposed methodology is presented in Figure 1 for one of four BMCGs defined by [4], which is explained in Section 3.2. The methodology is repeated for each BMCG.

During the development of business model theory, many scholars have appointed different BMCs. The finite number and definition of BMCs, in the present research, 


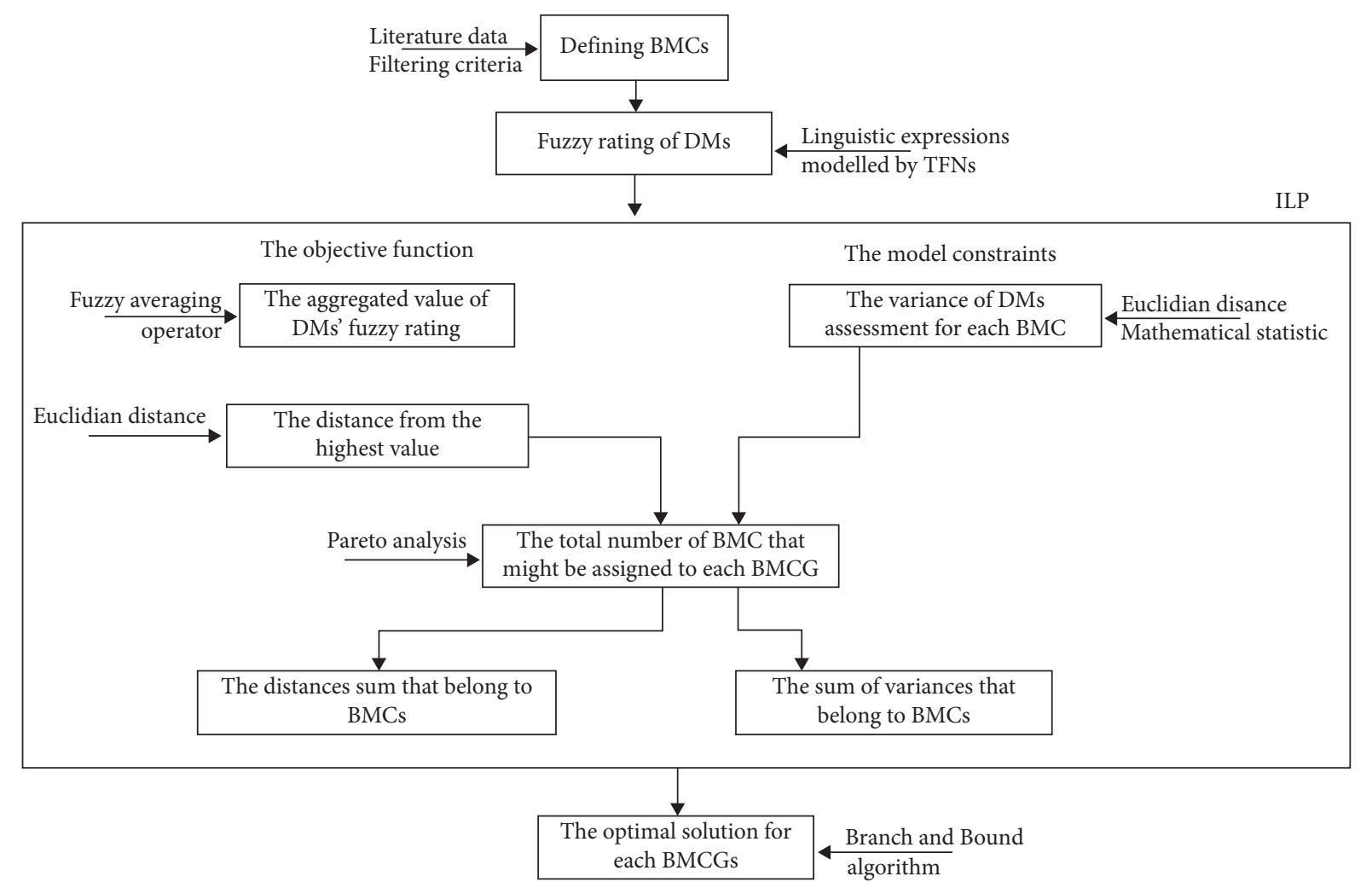

Figure 1: The model for BMCs' allocation to BMCGs.

start with the activities of literature data search and filtering criteria. At the same time, that finite number of identified BMCs should be adjoined to the four BMCGs defined by [4]. In the scope of the proposed research, the four economic sectors are considered (extraction, production, services, and research and development sector). The finite number of DMs is presented in Section 3.3. The DMs should assess the belonging of each identified BMC to the proposed BMCG by using predefined linguistic expressions. Since four groups of DMs corresponds to four different economic sectors, an aggregation procedure should be conducted.

At the level of each BMCG, the DMs are assessing the belonging of treated BMC. The first problem of the proposed methodology is to determine the total number of BMCs that should be assigned to each BMCG. This problem has been treated in literature in an almost negligible manner. Our research presumes that the total number of BMCs that might be assigned to each BMCG is defined by respecting the Pareto analysis. The second problem of the research is how to allocate the identified BMCs to BMCGs in an exact manner. For the course to a solution of this, the ILP model is defined to allocate each BMC to BMCG at the level of each BMCG. The ILP model consists of the objective function and the constraints.

The objective function is defined as a minimum of distances sum that belongs to BMCs derived as the output from the Pareto analysis. The distance from the aggregated value of DMs' fuzzy rating and the highest value that implies belief of certain belonging of BMC to BMCG is calculated.
In the scope of the proposed research, the errors of the DMs' assessment are presented by the variances. The variance of DMs assessment for each BMC at the level of each BMCG is determined. The model constraints are subject to the mean value of the variance of DMs assessment, which should be less than a predefined threshold value. The variance of BMCs that are considered for this calculation corresponds to the output from the Pareto analysis. As the consensus is reached when DMs use 3 consecutive expressions at most, those values are used for the calculation of variance, so the obtained value represents the threshold value.

The optimal solution for each BMCG content is obtained by the ILP model and Branch-and-Bound algorithm.

3.1. Definition of a Finite Set of BMCs. BMCs are formally represented as a set of indexes $\{1, \ldots, i, \ldots, I\}$. The total number of BMCs is designated $I$ and $i, i=1, . ., I$ is an index of BMC. Many studies are dealing with the problem of defining the BMs, which have been published in the last 20 years. By analyzing these studies, 317 BMCs have been identified. Considering the already mentioned issues, it could be noticed that some elements are very similar or very much the same, or many components which have been defined in similar ways have different titles. During the research, the technique of criteria filtering has been applied to consider the mentioned facts. The most important criteria stand if the proposed BMC is an integral part of other BMCs. In this way, the total number of 317 identified BMCs is decreased to 59 unique BMCs that are further considered in 
the research. To support the genuine of the identified BMCs, their explanation in the existing literature is presented in Table 1.

3.2. Definition of a Finite Set of BMCGs. BMCGs can be formally represented as a set of indexes $j=\{1, \ldots, j, \ldots, J\}$. A total number of BMCGs is designated $J$ and $j, j=1, . ., J$ is an index of BMCG. In this study, BMCGs are determined according to the author's suggestion, the reason being that it gives a very large distribution of terms that are used to define BM. These BMCGs are strategic choices $(j=1)$, value network $(j=2)$, creating value $(j=3)$, and capturing value $(j=4)$.

3.3. Definition of a Finite Set of DMs. Assessment of BMC $i, i=1 . ., I$ belonging to BMCG $j, j=1, . ., J$ on the scale of companies that belong to different economic sectors has been performed by the DMs. In this research, the DM is defined as the representative of the company from four different economic sectors in the Republic of Serbia. The competence of DMs is based on formal education and position in the company. The criterion of formal education is fulfilled if a person holds a master's degree or a higher-level degree. The criterion of position in a company, no matter if it is a private or public company, is fulfilled if a person is ranked at a senior management position or higher. Each of the four economics sectors has 10 representatives from different companies that participate in the panel discussion, which means 40 in total. Each BMC is assessed by different economic sector DMs, so $10 \mathrm{DMs}$ are bringing assessment during the panel discussion by using consensus. The DMs are formally presented by a set of indices $\varepsilon=\{1, \ldots, e, \ldots, E\}$. The total number of DMs is denoted as $E$, and $e, e=1, . ., E$ is an index of DM.

3.4. Choice of Appropriate Linguistic Variables for Describing the Values of BMCs. In this paper, it is assumed that the DMs expressed their assessments using one of the five predefined linguistic expressions. These linguistic expressions are modeled by TFNs:

It almost does not belong $(S 1)=(0,0,0.25)$.

Very small degree of belonging $(S 2)=(0.05,0.3,0.55)$.

Belongs spatially $(S 3)=(0.25,0.5,0.75)$.

Belongs significantly $(S 4)=(0.45,0.7,0.95)$.

Almost certainly belongs $(S 5)=(0.75,1,1)$.

Domains of these TFNs are defined in the real numbers set in the interval $[0,1]$. The value 0 and value 1 mark that the element $i, i=1, . ., I$ does not belong or that it fully belongs to the group $j, j=1, \ldots, J$, respectively.

The motivation for employment TFNs is supported by the fact that their usage does not demand complex mathematical operations. Simultaneously, the obtained solutions are accurate in a very sufficient manner taking into account the existing uncertainty in the treated problem.

\section{The Proposed Algorithm}

The algorithm is executed through the defined steps.

Step 1. A fuzzy rating of DMs can be presented:

$$
\widetilde{v}_{i j}^{e}=\left(l_{i j}^{e}, m_{i j}^{e}, u_{i j}^{e}\right) \text {. }
$$

Step 2. The aggregated value of DMs' fuzzy rating for each BMC $i, i=1, . ., I$ at the level of each BMCG $j, j=$ $1, . ., J$ is given by applying the fuzzy averaging method:

$$
\widetilde{v}_{i j}=\frac{1}{E} \cdot \sum_{e=1, . ., E} \widetilde{v}_{i j}^{e} .
$$

According to the rules of fuzzy algebra, $\widetilde{v}_{\mathrm{ij}}$ is TFN, too. Step 3. Let us calculate the variance of fuzzy rating of DMs for each BMC $i, i=1, . ., I$ at the level of each BMCG $j, j=1, . ., J$ :

$$
s_{i j}^{2}=\frac{1}{E-1} d^{2}\left(\widetilde{v}_{i j}^{e}, \widetilde{v}_{i j}\right) \text {. }
$$

Step 4. Let us set the ILP problem:

The objective function is as follows:

$$
\min _{i^{\prime}} \sum d\left(\widetilde{v}_{i j},(1,1,1)\right),
$$

for each $j, j=1, . ., J$,

where $d\left(\widetilde{v}_{i j},(1,1,1)\right)$ is calculated as the Euclidean distance between two TFNs [38].

The constraints are as follows:

$$
\frac{1}{I^{\prime}} \cdot \sum_{i=1, \ldots, I^{\prime}} s_{i j}^{2} \leq\left(\sigma^{2}\right)^{*} .
$$

$I^{\prime}$ is the total number of BMCs that are allocated to each BMCG by respecting the Pareto analysis.

The value of the right side of constraints $\left(\sigma^{2}\right)^{*}$ is defined as the variance threshold value of the fuzzy rating of DMs. It is a value of the variance where DMs reach consensus. In this research, an assumption is introduced that DMs are reaching consensus if three linguistic expressions in a row are used for fuzzy rating of DMs.

Step 5. By using the Gurobi solver, which is enhanced with the $\mathrm{B} \& \mathrm{~B}$ method, the arranged $I^{\prime}$ set of BMCs is sequentially introduced into BMCGs, the allocation of which is random.

\section{Case Study}

The input data for the proposed methodology is obtained through the online panel discussion. The period of conducting the research was 2020. As DMs are brought from four economic sectors, the ten DMs from each sector were put together to participate in the panel discussion. At the level of each group of ten panelists, the analysis of the finite list BMCs was performed. In compliance with the principles 
TABle 1: The review of BMCs according to literature data.

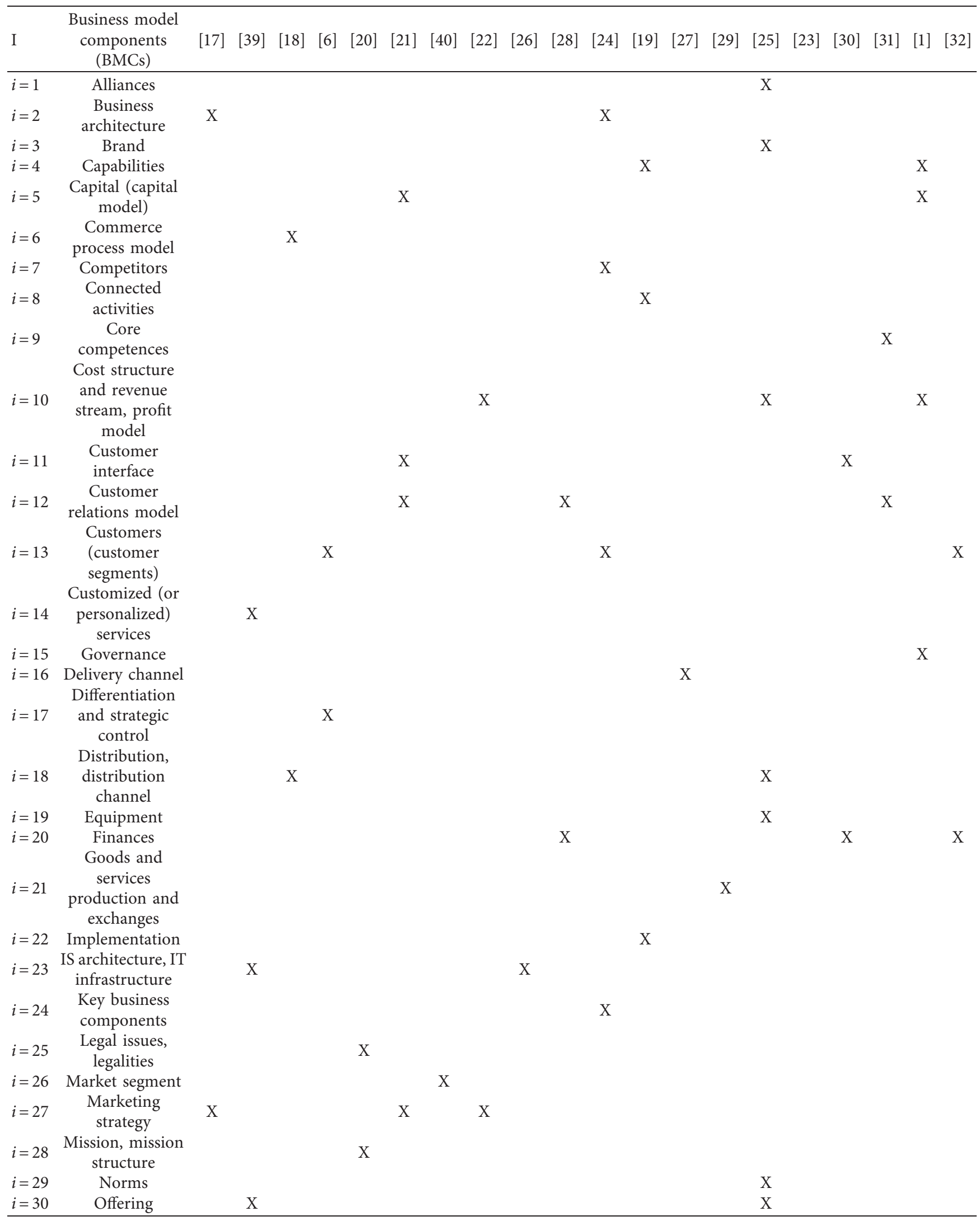


TABLE 1: Continued.

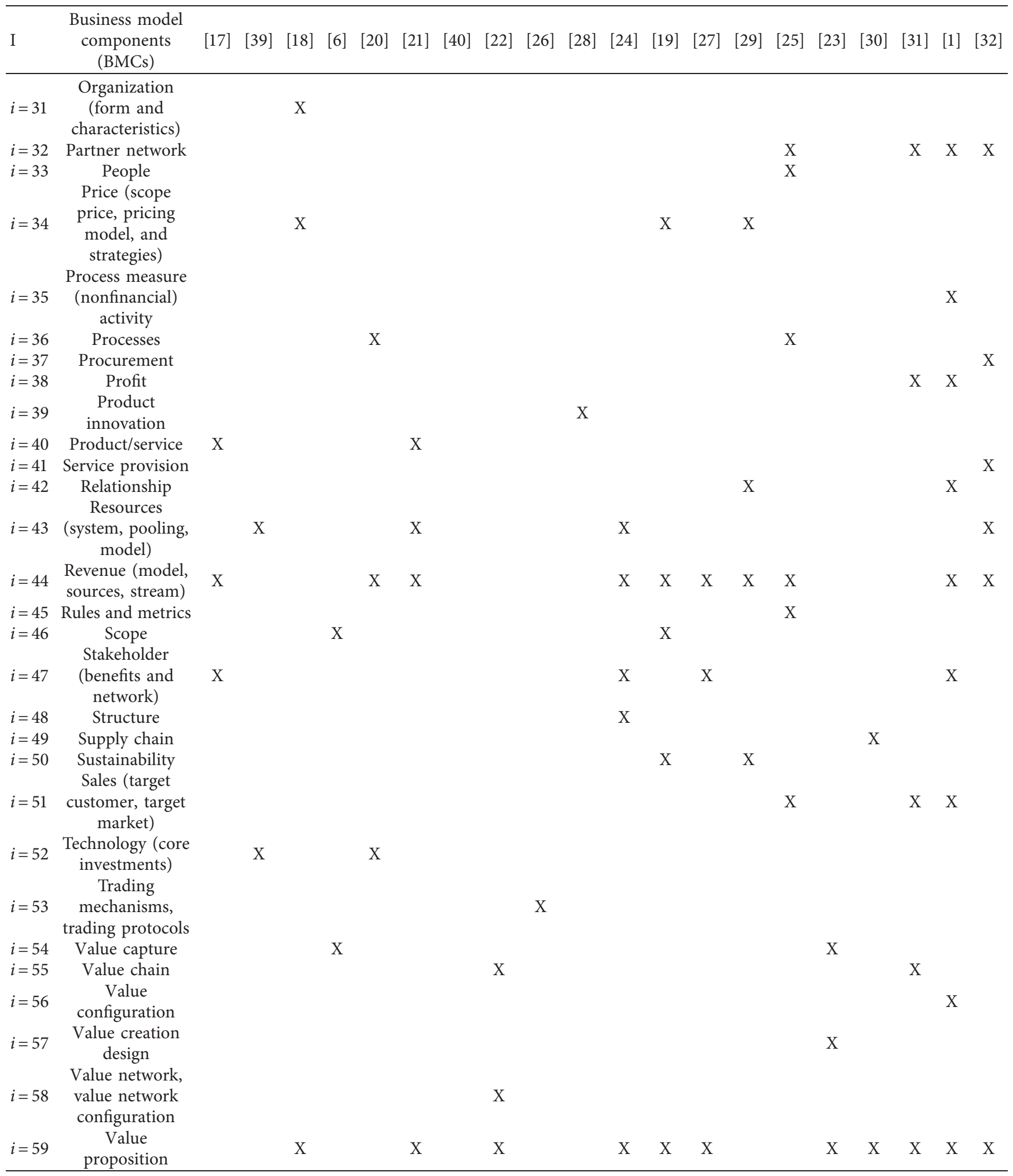

of the Affinity Diagram technique, each DMs' group had the goal to affiliate each of 59 BMCs to the 4 BMCGs, considering that each BMC may be affiliated to each BMCG. The decision was brought by using consensus. After the performed panel discussion, the collected data was used as input data for testing the proposed methodology.

Each group of ten DMs has performed independently as is defined in the proposed algorithm. Also, each group of 
DMs is supplied with the five predefined linguistic expressions to perform this assessment.

5.1. An Application of the Proposed Model. The algorithm is executed by following the procedure defined in Section 4 . The proposed procedure (Step 1 to Step 4 of the proposed algorithm) is illustrated by an example.

Let us assess the degree of belonging of BMC core competences $(i=9)$ to BMCG strategic choices $(j=1)$ :

$$
\begin{aligned}
& \widetilde{v}_{91}^{1}=S 3, \\
& \widetilde{v}_{91}^{2}=S 3, \\
& \widetilde{v}_{91}^{3}=S 5, \\
& \widetilde{v}_{91}^{4}=S 4 .
\end{aligned}
$$

The aggregated value of fuzzy rating of DMs presented for BMC $(i=9)$ and BMCG $(j=1)$ is

$$
\widetilde{v}_{91}=\frac{1}{4} \cdot\{(0.25,0.5,0.75)+(0.25,0.5,0.75)+(0.75,1,1)+(0.45,0.7,0.95)\}=(0.425,0.675,0.862) .
$$
that

The variance of fuzzy rating of DMs is stated as (4.3) so

$$
s_{91}^{2}=\frac{1}{4-1} \cdot\left\{\begin{array}{c}
\frac{1}{3} \cdot\left[(0.25-0.425)^{2}+(0.5-0.675)^{2}+(0.75-0.862)^{2}\right]+ \\
\frac{1}{3} \cdot\left[(0.25-0.425)^{2}+(0.5-0.675)^{2}+(0.75-0.862)^{2}\right]+ \\
\frac{1}{3} \cdot\left[(0.75-0.425)^{2}+(1-0.675)^{2}+(1-0.862)^{2}\right]+ \\
\frac{1}{3} \cdot\left[(0.45-0.425)^{2}+(0.7-0.675)^{2}+(0.95-0.862)^{2}\right]
\end{array}\right\}=0.129
$$

The distance between $\widetilde{v}_{91}$ and point $(1,1,1)$ is denoted as $d_{91}$. It is calculated:

$$
d_{91}=\sqrt{\frac{\left[(1-0.425)^{2}+(1-0.675)^{2}+(1-0.862)^{2}\right]}{3}}=0.39
$$

The aggregated values are calculated in a similar way, as well as variance and distances of the rest of BMCs at the level of considered BMCGs Table 2.

Based on the known data from the Pareto analysis, it is known that $20 \%$ of the considered items have the greatest importance for the considered problem. Respecting this fact, in this case, 12 BMCs best describe each BMCG. For this purpose, a model was developed whose application makes it possible to allocate BMCs to 4 BMCGs in the exact way as shown in Figure 2. By using Gurobi 9.1 (Step 5 of the proposed algorithm), the optimal solutions are found and presented.

In the scope of the proposed research, the Gurobi solver has employed a gap between the best and possible solutions. The threshold of $0,0 \%$ has been introduced, which provides the status of the optimal solution. The analysis of Figure 2 indicates that all four BMCGs have the same importance. In this way, different business models can be described by using the proposed BMCs with creating their descriptions and interactions.

5.2. The Discussion of the Results. As is well known, the use of BMs allows management to understand how it creates value for the customer and how it makes a profit. To understand it better, it is necessary to analyze each BMCG in more detail by using the appropriate BMCs (Figure 2).

The conducted analysis reveals that most of the treated BMCs are defined in Business Model Canvas except the component denoted as resources. This component is broken down into capital (including human capital and intellectual capital), finances, and equipment. In this way, scholars and companies might propose their own view of the business model framework and customize it for their own needs. 


\begin{tabular}{|l|}
\hline \multicolumn{1}{|c|}{ Strategic choices } \\
\hline Business architecture \\
Brand \\
Capital (capital model) \\
Commerce process model \\
Governance \\
Differentiation and strategic control \\
Market segment \\
Marketing strategy \\
Mission, mission structure \\
Organization (form and characteristics) \\
Scope \\
Sustainability
\end{tabular}

\begin{tabular}{|l|}
\cline { 2 - 2 } \multicolumn{1}{l|}{} \\
\hline Customer relations model \\
Customers (customer segments) \\
Delivery channel \\
Distribution, distribution channel \\
Goods and services production and exchanges \\
Partner network \\
Procurement \\
Service provision \\
Relationship \\
Stakeholder (benefits and network) \\
Supply chain \\
Value network, value network configuration \\
\hline
\end{tabular}

\begin{tabular}{|l|}
\hline \multicolumn{1}{|c|}{ Creating value } \\
\hline Capabilities \\
Connected activities \\
Equipment \\
Implementation \\
Process measure (nonfinancial) Activity \\
Processes \\
Product/service \\
Rules and metrics \\
Technology (core investments) \\
Value configuration \\
Value creation design \\
Value proposition \\
\hline
\end{tabular}

FIgUre 2: Allocation of BMCs to each BMCG.

TABLE 2: The variance and distances of the BMCs at the level of considered BMCGs.

\begin{tabular}{lccccccccccc}
\hline & Strategic choice & \multicolumn{3}{c}{ Value network } & \multicolumn{3}{c}{ Creating value } & \multicolumn{2}{c}{ Capturing value } \\
\hline & $d_{i 1}$ & $s_{i 1}^{2}$ & & $d_{i 2}$ & $s_{i 2}^{2}$ & & $d_{i 3}$ & $s_{i 3}^{2}$ & $d_{i 4}$ & $s_{i 4}^{2}$ \\
$i=1$ & 0.247 & 0.061 & $i=1$ & 0.288 & 0.134 & $i=1$ & 0.678 & 0.129 & $i=1$ & 0.824 & 0.141 \\
$i=2$ & 0.144 & 0.000 & $i=2$ & 0.774 & 0.134 & $i=2$ & 0.824 & 0.141 & $i=2$ & 0.677 & 0.301 \\
$i=3$ & 0.144 & 0.000 & $i=3$ & 0.678 & 0.129 & $i=3$ & 0.582 & 0.264 & $i=3$ & 0.535 & 0.264 \\
$i=4$ & 0.681 & 0.030 & $i=4$ & 0.475 & 0.395 & $i=4$ & 0.193 & 0.046 & $i=4$ & 0.582 & 0.292 \\
$i=5$ & 0.193 & 0.046 & $i=5$ & 0.725 & 0.268 & $i=5$ & 0.587 & 0.110 & $i=5$ & 0.824 & 0.141 \\
$i=6$ & 0.144 & 0.000 & $i=6$ & 0.725 & 0.268 & $i=6$ & 0.494 & 0.110 & $i=6$ & 0.725 & 0.268 \\
$i=7$ & 0.304 & 0.046 & $i=7$ & 0.464 & 0.566 & $i=7$ & 0.873 & 0.046 & $i=7$ & 0.569 & 0.566 \\
$i=8$ & 0.774 & 0.134 & $i=8$ & 0.582 & 0.292 & $i=8$ & 0.144 & 0.000 & $i=8$ & 0.678 & 0.209 \\
$i=9$ & 0.390 & 0.129 & $i=9$ & 0.475 & 0.395 & $i=9$ & 0.435 & 0.221 & $i=9$ & 0.678 & 0.129 \\
$i=10$ & 0.363 & 0.000 & $i=10$ & 0.774 & 0.134 & $i=10$ & 0.678 & 0.209 & $i=10$ & 0.144 & 0.000 \\
$i=11$ & 0.523 & 0.445 & $i=11$ & 0.823 & 0.061 & $i=11$ & 0.346 & 0.097 & $i=11$ & 0.416 & 0.495 \\
$i=12$ & 0.304 & 0.046 & $i=12$ & 0.193 & 0.046 & $i=12$ & 0.774 & 0.134 & $i=12$ & 0.774 & 0.134 \\
$i=13$ & 0.304 & 0.046 & $i=13$ & 0.193 & 0.046 & $i=13$ & 0.678 & 0.129 & $i=13$ & 0.678 & 0.129 \\
$i=14$ & 0.775 & 0.046 & $i=14$ & 0.523 & 0.445 & $i=14$ & 0.390 & 0.209 & $i=14$ & 0.575 & 0.296 \\
$i=15$ & 0.144 & 0.000 & $i=15$ & 0.774 & 0.296 & $i=15$ & 0.677 & 0.301 & $i=15$ & 0.823 & 0.061 \\
$i=16$ & 0.592 & 0.221 & $i=16$ & 0.193 & 0.046 & $i=16$ & 0.428 & 0.405 & $i=16$ & 0.824 & 0.141 \\
\hline
\end{tabular}


TABle 2: Continued.

\begin{tabular}{|c|c|c|c|c|c|c|c|c|c|c|c|}
\hline \multicolumn{3}{|c|}{ Strategic choice } & \multicolumn{3}{|c|}{ Value network } & \multicolumn{3}{|c|}{ Creating value } & \multicolumn{3}{|c|}{ Capturing value } \\
\hline$i=17$ & 0.144 & 0.000 & $i=17$ & 0.725 & 0.268 & $i=17$ & 0.729 & 0.000 & $i=17$ & 0.725 & 0.268 \\
\hline$i=18$ & 0.587 & 0.110 & $i=18$ & 0.193 & 0.046 & $i=18$ & 0.428 & 0.405 & $i=18$ & 0.774 & 0.134 \\
\hline$i=19$ & 0.435 & 0.221 & $i=19$ & 0.667 & 0.483 & $i=19$ & 0.288 & 0.134 & $i=19$ & 0.873 & 0.046 \\
\hline$i=20$ & 0.346 & 0.097 & $i=20$ & 0.873 & 0.046 & $i=20$ & 0.824 & 0.141 & $i=20$ & 0.193 & 0.046 \\
\hline$i=21$ & 0.727 & 0.152 & $i=21$ & 0.247 & 0.061 & $i=21$ & 0.247 & 0.061 & $i=21$ & 0.823 & 0.061 \\
\hline$i=22$ & 0.582 & 0.212 & $i=22$ & 0.725 & 0.268 & $i=22$ & 0.144 & 0.000 & $i=22$ & 0.823 & 0.061 \\
\hline$i=23$ & 0.390 & 0.209 & $i=23$ & 0.376 & 0.301 & $i=23$ & 0.540 & 0.160 & $i=23$ & 0.774 & 0.134 \\
\hline$i=24$ & 0.435 & 0.221 & $i=24$ & 0.677 & 0.301 & $i=24$ & 0.376 & 0.301 & $i=24$ & 0.582 & 0.292 \\
\hline$i=25$ & 0.247 & 0.061 & $i=25$ & 0.667 & 0.483 & $i=25$ & 0.824 & 0.141 & $i=25$ & 0.824 & 0.141 \\
\hline$i=26$ & 0.247 & 0.061 & $i=26$ & 0.630 & 0.221 & $i=26$ & 0.481 & 0.212 & $i=26$ & 0.535 & 0.264 \\
\hline$i=27$ & 0.231 & 0.141 & $i=27$ & 0.727 & 0.097 & $i=27$ & 0.449 & 0.120 & $i=27$ & 0.435 & 0.221 \\
\hline$i=28$ & 0.144 & 0.000 & $i=28$ & 0.824 & 0.141 & $i=28$ & 0.824 & 0.141 & $i=28$ & 0.774 & 0.134 \\
\hline$i=29$ & 0.273 & 0.296 & $i=29$ & 0.823 & 0.061 & $i=29$ & 0.571 & 0.395 & $i=29$ & 0.774 & 0.296 \\
\hline$i=30$ & 0.494 & 0.110 & $i=30$ & 0.587 & 0.030 & $i=30$ & 0.369 & 0.483 & $i=30$ & 0.288 & 0.134 \\
\hline$i=31$ & 0.193 & 0.046 & $i=31$ & 0.587 & 0.110 & $i=31$ & 0.727 & 0.097 & $i=31$ & 0.774 & 0.134 \\
\hline$i=32$ & 0.449 & 0.040 & $i=32$ & 0.144 & 0.000 & $i=32$ & 0.774 & 0.134 & $i=32$ & 0.774 & 0.296 \\
\hline$i=33$ & 0.331 & 0.188 & $i=33$ & 0.390 & 0.129 & $i=33$ & 0.428 & 0.405 & $i=33$ & 0.725 & 0.268 \\
\hline$i=34$ & 0.405 & 0.030 & $i=34$ & 0.873 & 0.046 & $i=34$ & 0.774 & 0.296 & $i=34$ & 0.144 & 0.000 \\
\hline$i=35$ & 0.634 & 0.120 & $i=35$ & 0.681 & 0.030 & $i=35$ & 0.193 & 0.046 & $i=35$ & 0.725 & 0.268 \\
\hline$i=36$ & 0.587 & 0.110 & $i=36$ & 0.727 & 0.097 & $i=36$ & 0.144 & 0.000 & $i=36$ & 0.678 & 0.209 \\
\hline$i=37$ & 0.727 & 0.097 & $i=37$ & 0.247 & 0.061 & $i=37$ & 0.416 & 0.495 & $i=37$ & 0.628 & 0.394 \\
\hline$i=38$ & 0.774 & 0.134 & $i=38$ & 0.725 & 0.188 & $i=38$ & 0.628 & 0.394 & $i=38$ & 0.144 & 0.000 \\
\hline$i=39$ & 0.390 & 0.129 & $i=39$ & 0.630 & 0.221 & $i=39$ & 0.369 & 0.483 & $i=39$ & 0.725 & 0.268 \\
\hline$i=40$ & 0.571 & 0.395 & $i=40$ & 0.405 & 0.030 & $i=40$ & 0.231 & 0.141 & $i=40$ & 0.540 & 0.160 \\
\hline$i=41$ & 0.774 & 0.134 & $i=41$ & 0.247 & 0.061 & $i=41$ & 0.346 & 0.129 & $i=41$ & 0.304 & 0.046 \\
\hline$i=42$ & 0.727 & 0.097 & $i=42$ & 0.144 & 0.000 & $i=42$ & 0.727 & 0.097 & $i=42$ & 0.582 & 0.292 \\
\hline$i=43$ & 0.288 & 0.134 & $i=43$ & 0.510 & 0.708 & $i=43$ & 0.449 & 0.040 & $i=43$ & 0.824 & 0.141 \\
\hline$i=44$ & 0.494 & 0.110 & $i=44$ & 0.571 & 0.395 & $i=44$ & 0.774 & 0.134 & $i=44$ & 0.310 & 0.531 \\
\hline$i=45$ & 0.435 & 0.141 & $i=45$ & 0.823 & 0.061 & $i=45$ & 0.247 & 0.061 & $i=45$ & 0.774 & 0.296 \\
\hline$i=46$ & 0.144 & 0.000 & $i=46$ & 0.475 & 0.395 & $i=46$ & 0.677 & 0.301 & $i=46$ & 0.677 & 0.301 \\
\hline$i=47$ & 0.405 & 0.030 & $i=47$ & 0.231 & 0.141 & $i=47$ & 0.678 & 0.129 & $i=47$ & 0.527 & 0.264 \\
\hline$i=48$ & 0.247 & 0.061 & $i=48$ & 0.582 & 0.212 & $i=48$ & 0.571 & 0.395 & $i=48$ & 0.873 & 0.046 \\
\hline$i=49$ & 0.727 & 0.097 & $i=49$ & 0.144 & 0.000 & $i=49$ & 0.449 & 0.120 & $i=49$ & 0.774 & 0.296 \\
\hline$i=50$ & 0.144 & 0.000 & $i=50$ & 0.678 & 0.129 & $i=50$ & 0.725 & 0.268 & $i=50$ & 0.873 & 0.046 \\
\hline$i=51$ & 0.346 & 0.129 & $i=51$ & 0.390 & 0.209 & $i=51$ & 0.630 & 0.221 & $i=51$ & 0.523 & 0.445 \\
\hline$i=52$ & 0.304 & 0.046 & $i=52$ & 0.774 & 0.134 & $i=52$ & 0.193 & 0.046 & $i=52$ & 0.540 & 0.080 \\
\hline$i=53$ & 0.523 & 0.365 & $i=53$ & 0.435 & 0.221 & $i=53$ & 0.678 & 0.209 & $i=53$ & 0.331 & 0.268 \\
\hline$i=54$ & 0.677 & 0.301 & $i=54$ & 0.824 & 0.141 & $i=54$ & 0.774 & 0.296 & $i=54$ & 0.144 & 0.000 \\
\hline$i=55$ & 0.523 & 0.445 & $i=55$ & 0.390 & 0.129 & $i=55$ & 0.369 & 0.483 & $i=55$ & 0.630 & 0.141 \\
\hline$i=56$ & 0.540 & 0.160 & $i=56$ & 0.678 & 0.209 & $i=56$ & 0.144 & 0.000 & $i=56$ & 0.824 & 0.141 \\
\hline$i=57$ & 0.582 & 0.292 & $i=57$ & 0.582 & 0.292 & $i=57$ & 0.144 & 0.000 & $i=57$ & 0.774 & 0.134 \\
\hline$i=58$ & 0.628 & 0.394 & $i=58$ & 0.144 & 0.000 & $i=58$ & 0.535 & 0.264 & $i=58$ & 0.824 & 0.141 \\
\hline$i=59$ & 0.569 & 0.566 & $i=59$ & 0.582 & 0.292 & $i=59$ & 0.193 & 0.046 & $i=59$ & 0.582 & 0.292 \\
\hline
\end{tabular}

From the managerial implications' perspective, it should be noticed that business product/service added value is crucial. Creating value is described by enabling conditions, value proposition and configuration, implementation, and establishing processes, so different strategic concepts may be further employed to derive the rest of business activities. Capturing value is described through core competences, revenue model, value chain, and other value and finance components. Above all, managers, while creating value, should create and maintain a value network. 


\section{Conclusion}

In the scope of the research, extensive literature regarding the BM domain is considered. By applying the criteria filtering technique, $59 \mathrm{BMCs}$ that are mostly used in literature have been identified. In the research, DMs from various industry companies have been assessing the significance of identified BMCs at the level of each BMCG. DMs used one of the five predefined linguistic expressions, which were modeled by TFNs. The assessment problem is stated as a fuzzy group decision-making problem. Since it is considered that all DMs have equal importance, the aggregated value of the fuzzy rating of DMs is given by using the fuzzy averaging operator.

Allocation problem is stated as ILP and appropriate BMCs at the level of each BMCG by using B\&B. In this way, the obtained solutions are less burdened with DMs prejudices than in papers that can be found in literature.

The contributions of this research could be denoted as theoretical. The contributions in the theoretical domain are as follows: (1) the analysis of BMCs that have been defined in the last 20 years and have been systematized and (2) methodological enhancement of affinity diagram. The enhancement of affinity diagram embraces (i) assessment of the belonging to each proposed group, which is based on usage of linguistic expressions by DMs, so it implies accurate assessment, (ii) modeling linguistic terms, which is based on fuzzy sets theory, and (iii) allocation of BMCs to BMCGs, which is determined in an exact way.

The proposed procedure can be used to analyze business enterprises that exist in different economic domains. This can be marked as the practical contribution of the study. The proposed methodology could be used for solving different management problems where the same relative importance of DMs is employed.

The main constraint of the proposed method is that DMs must have significant knowledge and experience in different areas to correctly conduct an assessment. As the model is large-sized, DMs need to spend significant time to complete the survey. Also, it is worth mentioning that each BMC is not uniquely defined as different scholars suggest the diverse scope of each BMC.

Future research should be focused on the determination of the relationship between BMCs under each BMCG. In this way, the improvement of BMs may be achieved by enhancing business processes derived from BMCs, or through the reengineering of the BMs by applying different frameworks. The interaction of the business processes could be performed through different analyses, such as as-is process analysis.

\section{Data Availability}

All data generated or analyzed during this study are included in this paper.

\section{Conflicts of Interest}

The authors declare no conflicts of interest.

\section{References}

[1] A. Upward and P. Jones, “An ontology for strongly sustainable business models: defining an enterprise framework compatible with natural and social science," Organization \& Environment, vol. 29, no. 1, pp. 97-123, 2015.

[2] T. Ritter and C. Lettl, "The wider implications of businessmodel research," Long Range Planning, vol. 51, no. 1, pp. 1-8, 2018.

[3] M. M. Al-Debei and D. Avison, "Developing a unified framework of the business model concept," European Journal of Information Systems, vol. 19, no. 3, pp. 359-376, 2010.

[4] S. M. Shafer, H. J. Smith, and J. C. Linder, "The power of business models," Business Horizons, vol. 48, no. 3, pp. 199-207, 2005.

[5] Y. Zhang, S. Zhao, and X. Xu, "Business model innovation: an integrated approach based on elements and functions," Information Technology and Management, vol. 17, no. 3, pp. 303-310, 2015.

[6] D. W. Stewart and Q. Zhao, "Internet marketing, business models, and public policy," Journal of Public Policy and Marketing, vol. 19, no. 2, pp. 287-296, 2018.

[7] S. Widjajanto and E. Rimawan, "Modified failure mode and effect analysis approaching to improve organization performance based on baldrige criteria - a case study of an electromedic industry," Operational Research in Engineering Sciences: Theory and Applications, vol. 4, no. 3, pp. 39-58, 2021.

[8] H.-J. Zimmermann, "Fuzzy set theory," Wiley Interdisciplinary Reviews: Computational Statistics, vol. 2, no. 3, pp. 317-332, 2010.

[9] W. He, R. M. Rodríguez, B. Dutta, and L. Martínez, “A type-1 OWA operator for extended comparative linguistic expressions with symbolic translation," Fuzzy Sets and Systems, 2021, in Press.

[10] S. Nestic, J. F. Lampón, A. Aleksic, P. Cabanelas, and D. Tadic, "Ranking manufacturing processes from the quality management perspective in the automotive industry," Expert Systems, vol. 36, no. 6, p. e12451, 2019.

[11] D. Tadic, A. Aleksic, P. Mimovic, H. Puskaric, and M. Misita, "A model for evaluation of customer satisfaction with banking service quality in an uncertain environment," Total Quality Management and Business Excellence, vol. 29, no. 11-12, pp. 1342-1361, 2016.

[12] E.-G. Talbi, Metaheuristics: From Design to Implementation, John Wiley \& Sons, Hoboken, NJ, USA, 2009.

[13] B. Khara, J. K. Dey, and S. K. Mondal, "An integrated imperfect production system with advertisement dependent demand using branch and bound technique," Flexible Services and Manufacturing Journal, vol. 33, no. 2, pp. 508-546, 2021.

[14] O. Ozturk, M. A. Begen, and G. S. Zaric, "A branch and bound algorithm for scheduling unit size jobs on parallel batching machines to minimize makespan," International Journal of Production Research, vol. 55, no. 6, pp. 1815-1831, 2016.

[15] M. Tamannaei and I. Irandoost, "Carpooling problem: a new mathematical model, branch-and-bound, and heuristic beam search algorithm," Journal of Intelligent Transportation Systems, vol. 23, no. 3, pp. 203-215, 2018.

[16] R. Walter and P. Schulze, "On the performance of task-oriented branch-and-bound algorithms for workload smoothing in simple assembly line balancing," International Journal of Production Research, pp. 1-14, 2021.

[17] P. Timmers, "Business models for electronic markets," Electronic Markets, vol. 8, no. 2, pp. 3-8, 1998. 
[18] J. Linder and S. Cantrell, Changing Business Models: Surveying the LandscapeAccenture Institute for Strategic Change, Cambridge, UK, 2000.

[19] A.. Afuah, Business Models: A Strategic Management Approach, McGraw-Hill/Irwin, New York, NY, USA, 2004.

[20] R. Alt and H.-D. Zimmermann, "Preface: introduction to special section - business models," Electronic Markets, vol. 11, no. 1, pp. 3-9, 2001.

[21] O. Petrovic, C. Kittl, and R. D. Teksten, "Developing business models for ebusiness," SSRN Electronic Journal, 2001.

[22] H. Chesbrough, R. S. Rosenbloom, and J. S. Brown, "The role of the business model in capturing value from innovation: evidence from xerox corporation's technology spinoff companies," Industrial and Corporate Change, vol. 11, pp. 529$555,2002$.

[23] J. Richardson, "The business model: an integrative framework for strategy execution," Strategic Change, vol. 17, no. 5-6, pp. 133-144, 2008.

[24] J. Hedman and T. Kalling, "The business model concept: theoretical underpinnings and empirical illustrations," European Journal of Information Systems, vol. 12, no. 1, pp. 49-59, 2003.

[25] M. W. Johnson, C. M. Christensen, and H. Kagermann, "Reinventing your business model," Harvard Business Review, vol. 86, pp. 50-59, 2008.

[26] K. Lyytinen and S. McGann, "Capturing the dynamics of eBusiness models: the eBusiness analysis framework and the electronic trading infrastructure," in Proceedings of the 15th Annual Bled Electronic Commerce Conference, pp. 36-54, Bled, Slovenia, 2002.

[27] A. Osterwalder and Y. Pigneur, "15th bled electronic commerce conference e-reality: constructing the e-economy an e-business model ontology for modeling e-business," in Proceedings of the Electronic Commerce Conference - EReality: Constructing the EEconomy, pp. 75-91, Bled, Slovenia, 2002.

[28] A. Osterwalder and Y. Pigneur, "An ontology for e-business models," in Value Creation from E-Business Models, W. Currie, Ed., pp. 65-97, Butterworth-Heinemann, Lausanne, Switzerland, 2003.

[29] E. Brousseau and T. Penard, "The economics of digital business models: a framework for analyzing the economics of platforms," Review of Network Economics, vol. 6, no. 2, pp. 81-114, 2007.

[30] F. Boons and F. Lüdeke-Freund, "Business models for sustainable innovation: state-of-the-art and steps towards a research agenda," Journal of Cleaner Production, vol. 45, pp. 9-19, 2013.

[31] Y. Taran, H. Boer, and P. Lindgren, "A business model innovation typology," Decision Sciences, vol. 46, no. 2, pp. 301-331, 2015.

[32] B. W. Wirtz, A. Pistoia, S. Ullrich, and V. Göttel, "Business models: origin, development and future research perspectives," Long Range Planning, vol. 49, no. 1, pp. 36-54, 2016.

[33] D. Tadić, S. Arsovski, A. Aleksić, M. Stefanović, and S. Nestić, "A fuzzy evaluation of projects for business processes' quality improvement," Intelligent Systems Reference Library, vol. 87, pp. 559-579, 2015.

[34] Z. Ali, T. Mahmood, T. Mahmood, K. Ullah, and Q. Khan, "Einstein geometric aggregation operators using a novel complex interval-valued pythagorean fuzzy setting with application in green supplier chain management," Reports in Mechanical Engineering, vol. 2, no. 1, pp. 105-134, 2021.
[35] J. Bukchin and J. Rubinovitz, "A weighted approach for assembly line design with station paralleling and equipment selection," IIE Transactions, vol. 35, pp. 73-85, 2003.

[36] B. Mayer, M. Killian, and M. Kozek, "A branch and bound approach for building cooling supply control with hybrid model predictive control," Energy and Buildings, vol. C, no. 128 , pp. 553-566, 2016.

[37] R. Yokoyama, Y. Shinano, Y. Wakayama, and T. Wakui, "Model reduction by time aggregation for optimal design of energy supply systems by an MILP hierarchical branch and bound method," Energy, vol. 181, pp. 782-792, 2019.

[38] P. Grzegorzewski, "Distances between intuitionistic fuzzy sets and/or interval-valued fuzzy sets based on the Hausdorff metric," Fuzzy Sets and Systems, vol. 148, no. 2, pp. 319-328, 2004.

[39] S. Bagchi and B. Tulskie, "E-Business models: integrating learning from strategy development experiences and empirical research," in Proceedings of the 20th Annual International Conference of the Strategic Management Society, pp. 15-18, Vancouver, Canada, 2000.

[40] J. F. Rayport and B. J. Jaworski, Introduction to E-Commerce, McGraw-Hill Irwin MarketspaceU, Boston, MA, USA, 2004. 